\title{
Advances in Ablation of Ventricular Tachycardia in Nonischemic Cardiomyopathy
}

\author{
Nilesh Mathuria, $\mathbf{M D}^{1,2}$, Roderick Tung, $\mathbf{M D}^{3}$, and Kalyanam Shivkumar, MD, $\mathbf{P h D}^{3}$ \\ ${ }^{1}$ St. Luke's Episcopal Hospital/Texas Heart Institute, Houston, TX \\ 2University of Texas at Houston Health Science Center, Houston, TX \\ ${ }^{3}$ UCLA Cardiac Arrhythmia Center, David Geffen School of Medicine at UCLA Los Angeles, CA
}

\section{Abstract}

Management of patients with nonischemic cardiomyopathy (NICM) and ventricular tachycardia (VT) remains challenging. The role of catheter ablation for VT continues to evolve for these patients. Prior reports have described the location of the arrhythmogenic substrate for patients with NICM to be frequently located along the basal left ventricle, with an epicardial predilection. Additionally, predictors for identifying whether mapping the endocardium or epicardial surface of the heart have been identified for improved success of VT ablation in this patient population. This chapter will review the latest advances in catheter ablation of ventricular tachycardia in patients with NICM.

\section{Keywords}

Catheter ablation; Epicardial; Electroanatomic mapping; Nonischemic Cardiomyopathy; Ventricular Tachycardia

\section{Introduction}

Patients with prior myocardial infarction have been known to have reentrant ventricular tachycardia (VT) due to myocardial scar. While the approach to patients with nonischemic cardiomyopathy (NICM) has been translated from the post-infarct substrate, our understanding of the NICM arrhythmogenic substrate continues to evolve. Advances in electroanatomic mapping, cardiac imaging, and epicardial access have improved ablation strategies. The objective of this chapter is to provide an update regarding advances in catheter ablation for ventricular tachycardia in NICM.

\section{Location of the Arrhythmogenic Substrate}

Analysis of explanted human hearts in patients with dilated cardiomyopathy has revealed multiple abnormalities. Initial observations of interstitial and replacement fibrosis were noted in autopsy series ${ }^{1,2}$. Additionally, necropsy analysis of 142 patients with dilated cardiomyopathy revealed grossly visible scar in the lateral left ventricle and frequently

\footnotetext{
Address for Correspondence: Kalyanam Shivkumar MD, PhD, UCLA Cardiac Arrhythmia Center, Ronal Reagan UCLA Medical Center, 47-123 CHS, David Geffen School of Medicine at UCLA, 10833 Le Conte Avenue, Los Angeles CA 90095-1679, USA, kshivkumar@mednet.ucla.edu.

Disclosure

No potential conflict of interest relevant to this article was reported.
} 
within the septum in $14 \%{ }^{3}$. In this same series, $35 \%$ and $57 \%$ of right and left ventricle sections, respectively, had evidence of replacement fibrosis during histological analysis ${ }^{3}$.

Aside from the presence of fibrosis and scar, electrical conduction properties are significantly altered in patients with dilated cardiomyopathy. Human tissue experiments from patients with NKICM have shown areas of fibrosis lead to fractionated electrograms and that myocardial conduction was delayed due to a change in activation around these

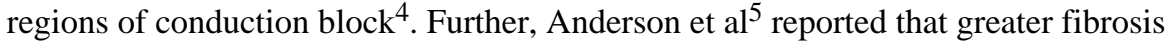
correlated with a higher level of conduction abnormalities. Based on this initial data, it is felt that the substrate for ventricular tachycardia in patients with NICM includes fibrosis with creation of areas of conduction block, conduction slowing around these regions which leads to anisotropic conduction and creates the nidus for reentrant ventricular tachycardia.

Unlike ischemic cardiomyopathy, where regions of scar correlate with regions of prior infarction, NICM does not follow a physiologic pattern. With the advent of electro-anatomic mapping ${ }^{6}$, a relatively non-invasive method could be used to assess regions of low voltage, which correlate with myocardial scar.

In 2003, Hsia et $\mathrm{al}^{7}$ described the endocardial substrate in patients with NICM and monomorphic VT. All 19 patients studied had evidence of basal, perivalvular low voltage consistent with scar. More importantly, $88 \%$ of all VT induced in this cohort emanated from the basal LV, suggesting this low voltage region is the critical substrate for VT in this patient population. Although the epicardial surface was only mapped in three patients, findings of abnormal electrograms and low voltage regions were noted, suggesting an epicardial substrate may co-exist in certain patients ${ }^{7}$.

In 2004, Soejima et $\mathrm{al}^{8}$ further described the arrhythmogenic substrate in patients with NICM undergoing VT ablation. Twenty patients underwent endocardial mapping which revealed evidence of scar in all patients $(\unlhd .5 \mathrm{mV})$, with the majority $(63 \%)$ of patients having a basal, perivalvular extent of scar. Furthermore, based on entrainment and pacemapping, $60 \%$ of patients had an identifiable endocardial isthmus which was targeted for ablation. Seven patients in this series underwent epicardial access and mapping after failed endocardial ablation. All seven patients had evidence of epicardial scar from the basal/lateral LV, RVOT, or basal RV. Additionally, 5/7 patients had both endocardial and epicardial scar with epicardial scar area being larger for these patients. Aside from scar, 6/7 patients had an epicardial VT isthmus identified which was targeted for ablation ${ }^{8}$. This publication confirmed the predilection of endocardial basal LV scar, but also suggested that an equally important epicardial substrate may be present and should be considered as an ablation target in patients with VT and NICM.

The importance of the epicardial substrate in NICM was further studied and delineated by Cano et $\mathrm{al}^{9}$ in 2009 (Fig. 1). In this study, 22 patients with either EKG criteria suggesting an epicardial exit or failed prior endocardial ablation were evaluated. Based on electroanatomic mapping, $82 \%$ of patients had evidence of epicardial scar with $77 \%$ having greater epicardial scar area compared to the endocardium. In addition to an epicardial scar, the location in $>70 \%$ of patients involved the basal LV extending to the lateral wall ${ }^{9}$. Of the 18 patients with an epicardial VT identified by entrainment mapping and/or pacemapping, $14 / 18$ patients were free from VT recurrence at followup of $18 \pm 7$ months 9 .

Although the above mentioned publications seem to imply patients with VT and NICM have a basal/lateral LV scar, other regions have been described. A recent report by Yokokawa et a ${ }^{10}$ described a series of 33 patients with NICM, of which, $24 \%$ had a VT originating from the aortic cusps. All patients with cusp VT $(n=8)$ had evidence of basal LV low voltage on EAM, and $75 \%$ of patients with LV mapping had a basal LV low voltage region. Of note, 
despite successful ablation within the aortic cusps, 7/8 patients with an aortic cusp VT had other VT induced during the procedure with $4 / 8$ patients having another VT recurrence during a mean followup period of 23 months ${ }^{10}$. Furthermore, 2/4 patients with recurrence required epicardial ablation to eliminate other VTs. This publication highlights that the typical basal LV endocardial arrhythmogenic substrate can extend into the aortic cusps and should be considered in patients with NICM and an inferior axis VT.

All the previously described publications regarding VT ablation in this patient population have noted a basal perivalvular LV substrate. Recently, however, the characteristics of patients with NICM and an isolated septal substrate have been reported ${ }^{11}$. Over an eleven year period at a single center, $11.6 \%$ (31/266) of patients with NICM undergoing VT ablation had an isolated septal substrate as defined abnormal bipolar $(<1.5 \mathrm{mV})$ or unipolar $(<8.3 \mathrm{mV})$ electrograms. These patients frequently had both LBBB and RBBB VT morphologies with ablation needing to be performed in both the RV and LV septum. Additionally, acute procedural non-inducibility was noted in only $66 \%$ and there was a $32 \%$ recurrence during a mean followup of 20 months. Of note, 14 patients also underwent epicardial mapping in this series, and only 5 patients had an epicardial low voltage area which was confined to the pre-aortic region of the LV summit ${ }^{11}$. This publication highlights the prevalence, characteristics, and course of patients with NICM and VT with an isolated septal arrhythmogenic substrate. This should be considered in patients with both LBBB and RBBB VT morphologies.

In addition to electroanatomic differences in patients with NICM, electrogram characteristics have also been studied and characterized in this patient population. In 2006, Cesario et $\mathrm{al}^{12}$ studied a series of 20 patients, of which 8 patients had NICM, that underwent endocardial and epicardial mapping for untolerated VT. Of the 8 patients with NICM, 2/8 required epicardial ablation. The ablation strategy chosen for these patients was solely substrate based targeting regions of slow conduction within scar and/or border zones of scar with appropriate pacemaps. As with prior studies, both the endocardium and epicardial surfaces had evidence of scar, yet there was no correlation with corresponding regions of scar endocardially vs epicardially. Further, two patients underwent cardiac transplantation and, surprisingly, regions of low voltage did not correlate with scar grossly. Although this was only with two patients, it suggested a more complex and poorly understood scar biology of patients with NICM.

We further characterized the arrhythmogenic substrate in NICM with regard to abnormal electrograms and late potentials. Late potentials were defined as electrograms which started after the QRS and are surrogates for evidence of slow conduction ${ }^{13}$. In 16 patients with NICM, there was a similar area of low voltage in both the endocardium and epicardium based on EAM. With ultra high-density mapping, there was a significantly lower number of late potentials and very late potentials ( $>100 \mathrm{~ms}$ post QRS) in the NICM cohort when compared to the ICM patients. Additionally, an ablation strategy targeting LPs was highly successful in the ICM patients (82\% freedom from VT at 12 month followup) yet the NICM cohort only had a 50\% freedom from VT at 15 months ${ }^{13}$. Consistent with these observations, Kuhne et $\mathrm{al}^{14}$ also assessed the prevalence of late potentials in patients with NICM undergoing VT ablation and noted a marked improvement in outcomes $(67 \%$ vs $7 \%$ freedom from VT) in patients with late potentials identified versus those without. The differences in late potential characterization seen between the ICM and NICM groups once again highlight the differences in scar biology in patients with NICM and reinforce the notion that current ablation strategies remain suboptimal. 


\section{Advances in Identifying the Epicardial Substrate}

The growing dataset shows that patients with NICM have both an endocardial and epicardial arrhythmogenic substrate, and epicardial substrate is often more extensive. Recent publications suggest the surface EKG, pre-procedure imaging, as well as electro-anatomic mapping may aid in predicting an epicardial substrate.

\section{2 lead EKG}

As with any electrophysiologic procedure, the 12 lead EKG provides invaluable information regarding the arrhythmia mechanism and/or location. A recent publication has suggested even the sinus EKG may predict a basal/lateral LV arrhythmogenic substrate in NICM. Tzou et al ${ }^{15}$ compared the sinus EKG of patients with NICM who had known VT versus those without VT or known scar. Their findings suggest that patients with NICM with a V1 Rwave $\searrow 0.15 \mathrm{mV}$, V6 Swave $\searrow 0.15 \mathrm{mV}$, and/or a V6 S:Rwave ratio of $\searrow 0.20 \mathrm{mV}$ predicted a basal/lateral LV low voltage on electro-anatomic mapping ${ }^{15}$. A major limitation was that not all patients without these EKG findings underwent imaging to assess for scar, nevertheless, this publication highlights even the sinus EKG may provide clues to the arrhythmogenic substrate in NICM.

Regarding epicardial versus endocardial myocardial activation, a greater percentage of the QRS is activated with direct myocardial conduction as opposed to via the His Purkinje system. This, in theory, would lead to greater slurring and delay in the initial upstroke of the QRS. This hypothesis was formally tested by Buerrezo et $\mathrm{al}^{16}$ where endocardial and epicardial pacing QRS morphologies were compared and these findings were correlated to patients that required epicardial ablation. Consistent with the theory of initial QRS "slurring" due to epicardial initiation, four distinct parameters favored an epicardial VT. This included a pseudo-delta wave of $234 \mathrm{~ms}$, an intrinsicoid deflection time in V2 $285 \mathrm{~ms}$, RS complex duration of $\geq 121 \mathrm{~ms}$, and total QRS duration. Although there was a greater QRS duration in epicardial VT, the first three parameters studied were more specific for an epicardial exit site ${ }^{16}$. This publication strongly suggested surface EKG features could predict an endocardial vs epicardial exit site VT. In 2006, Daniels et a ${ }^{17}$ described a novel maximal deflection index (MDI) to predict an epicardial exit site for VT arising from the sinus of Valsalva in patients with structurally normal heart. The MDI is the shortest time to maximal deflection in the precordial leads divided by the total QRS duration ${ }^{17}$. A value of $\searrow 0.55$ had a $100 \%$ sensitivity and a $98 \%$ specificity for an epicardial focus ${ }^{17}$.

Bazan et al assessed differences in EKG pattern in the endocardium vs epicardial surface in patients without prior myocardial infarction. Pacemaps from the basal superior and apicalsuperior epicardial LV had a q wave in lead 1 in $90 \%$ and $88 \%$ of patients, respectively ${ }^{18}$. Conversely, the basal inferior and apical inferior epicardial pacemaps had q waves in the inferior leads in $81 \%$ and $92 \%$ of patients, respectively ${ }^{18}$. Finally, in 2009 , Valles et al ${ }^{18}$ further studied endocardial vs epicardial exit sites in patients with NICM. Their results suggested a pseudo-delta $\geq 75 \mathrm{~ms}$ and a MDI $\searrow 0.59$ were more sensitive and specific for an epicardial VT. Further, a four-step algorithm was created which incorporated the presence or absence of q waves in lead 1 and/or the inferior leads. Given the basal/lateral predilection of VT in NICM, most VT would have a negative QRS in lead 1. If, however, the exit is epicardial then a qS pattern would be suggested versus an $\mathrm{rS}$ pattern with initial endocardial activation in this series (SN/SP 88\%). Further, in the inferior leads, a q wave did not suggest an epicardial exit implying initial endocardial activation away from the inferior leads ${ }^{19}$. With this data, a four step algorithm was created to determine if a VT was epicardial in patients with NICM with a sensitivity and specificity of $96 \%$ and $93 \%$, respectively ${ }^{19}$. First, the presence of $q$ waves in the inferior leads suggests an endocardial VT. Second, a pseudo- 
delta of $\geq 75 \mathrm{~ms}$ suggests an epicardial exit. Third, a MDI $\searrow 0.59$ suggest an epicardial exit. Finally, a qS in lead 1 predicted an epicardial exit for a $\mathrm{VT}^{19}$.

All the various publications discussed imply an epicardial exit would have a longer time before the His-Purkinje system was activated as evidenced by greater initial slurring of the QRS or greater time to the maximal deflection. Further, pacemaps and VT comparison also show lead specific criteria may aid in VT exit site determination. Although, antiarrhythmic drugs were discontinued for nearly all patients, other factors could contribute to these findings such as heart geometry and VT cycle length. Additionally, although exit sites may be predicted with these various parameters, the critical isthmus of these re-entrant VTs, may be ablated from the endocardium rather than the epicardium. Regardless, the clues that have been determined for potential epicardial exits remain invaluable in the planning for VT ablation in patients with NICM.

\section{Imaging}

In addition to the surface EKG, pre-procedural MR imaging has been shown to aid in identification of the arrhythmogenic substrate in NICM. In 2005, Nazarian et $\mathrm{al}^{20}$ reported the role of MRI in NICM and predicting VT during programmed stimulation. In summary, 26 patients underwent MRI prior to EPS. The transmural extent of scar was assessed in all patients. Patients with $25 \%-75 \%$ transmural extent of scar predicted inducible $\mathrm{VT}^{20}$. This was the first study to correlate the location and extent of scar with arrhythmogenic risk in patients with NICM. Assomull et al ${ }^{21}$ assessed the prognostic significance of "mid-wall fibrosis" noting a significant increased risk in sudden cardiac death and/or VT in patients with "mid-wall fibrosis." ${ }^{21}$. Aside from DHE on MRI predicting risk for VT, Bogun et al ${ }^{22}$ in 2009 demonstrated location of scar on MRI can also predict successful sites of ablation. In this study, 29 patients with NICM underwent pre-procedure MRI. 14/29 patients had DHE on MRI. 5 patients had predominant endocardial scar, and endocardial ablation was successful in ablation of VT. 2 patients, however, had an epicardial scar and epicardial mapping and ablation was successful in these patients. Finally, in the remainder of patients with intramural scar and/or extension to the endocardium had either partial or no success in ablation for $\mathrm{VT}^{22}$. This study allowed for the role of pre-procedure MR imaging to guide planning for ablation and potentially predict if epicardial mapping is warranted.

Aside from pre-procedure imaging, real time intra-procedural intracardiac echocardiography (ICE) has a role to assess for an epicardial substrate. Bala et $\mathrm{al}^{23}$ studied eighteen patients with NICM undergoing VT ablation who had an abnormal echogenicity in the lateral LV on ICE. All 18 patients had regions of epicardial low voltage on electroanatomic mapping that correlated with ICE, while only $50 \%$ of the patients had endocardial scar ${ }^{23}$. Although only one region of the LV was imaged in a limited number of patients, this publication highlights the potential of ICE technology for guiding VT ablation in patients with NICM.

\section{Electroanatomic Mapping}

Recent data now suggest intra-procedural electro-anatomic mapping characteristics may predict an epicardial arrhythmogenic substrate for patients with NICM. Hutchinson et $\mathrm{al}^{24}$ assessed the role of unipolar recordings during voltage maps created during VT ablation. In 11 patients with epicardial low voltage regions noted during epicardial mapping, 9/11 patients had corresponding low voltage ( $\$ 8.27 \mathrm{mV}$ ) unipolar recordings from the endocardial surface $^{24}$. This data suggested that unipolar recordings may have a greater field of view which could predict an epicardial substrate during endocardial mapping. This same methodology was used to assess the RV epicardium in patients with ARVD with an endocardial unipolar voltage of $<5.5 \mathrm{mV}$ indicative of an epicardial low voltage region ${ }^{25}$. 


\section{Endocardial/Epicardial or Both?}

Based on the above data, the question still remains as to the approach for VT ablation in patients with NICM. Multiple studies assessing the substrate and different ablation strategies in NICM continue to have less than optimal long-term outcomes (Table 1). Additionally, outcomes do not seem to be dramatically different when epicardial mapping is performed in addition to endocardial mapping. This, however, is based on small numbers of patients with an evolving understanding of the arrhythmogenic substrate of NICM. Further studies are needed to assess if initial combined endocardial/epicardial mapping leads to better outcomes in this patient population. Finally, a recent publication has suggested that surgical resection/ ablation can be considered in patients with NICM when endocardial and/or percutaneous epicardial mapping have failed ${ }^{26}$. This single center experience described eight patients with failed endocardial and/or epicardial ablation that underwent open surgical cryoablation. 75\% of the patients had a reduction in VT burden, while the remainder of patients died of nonarrhythmic events ${ }^{26}$. This report suggests that an open surgical ablation approach may be considered in select cases for patients with refractory VT.

When considering epicardial mapping, complications include RV puncture ${ }^{27}$, coronary arterial or venous injury, hemopericardium ${ }^{28}$, liver laceration, diaphragmatic bleeding, or phrenic nerve injury ${ }^{29}$. These potential complications highlight the need for continued improvement in technology for epicardial access as well as careful consideration of the patient characteristics prior to any planned procedure.

\section{Conclusions}

Tremendous knowledge has been gained in recent years regarding our understanding of VT in patients with NICM. This includes identifying the arrhythmogenic substrate, assessing the need for epicardial mapping, and advances in ablation technology. However, ablation outcomes are not as favorable as in patients with ICM and recurrences still occur despite ablation on both surfaces. This would indicate more work needs to be done in assessing and targeting intramural circuits, understanding scar biology in NICM and potentially targeting the autonomic nervous system to the heart ${ }^{30}$ which may improve outcomes for this patient population.

\section{References}

1. Unverferth DV, Baker PB, Swift SE, Chaffee R, Fetters JK, Uretsky BF, et al. Extent of myocardial fibrosis and cellular hypertrophy in dilated cardiomyopathy. Am J Cardiol. 1986; 57(10):816-820. [PubMed: 2938462]

2. Sugrue DD, Holmes DR Jr, Gersh BJ, Edwards WD, McLaran CJ, Wood DL, et al. Cardiac histologic findings in patients with life-threatening ventricular arrhythmias of unknown origin. $\mathrm{J}$ Am Coll Cardiol. 1984; 4(5):952-957. [PubMed: 6491087]

3. Roberts WC, Siegel RJ, McManus BM. Idiopathic dilated cardiomyopathy: analysis of 152 necropsy patients. Am J Cardiol. 1987; 60(16):1340-1355. [PubMed: 3687784]

4. de Bakker JM, van Capelle FJ, Janse MJ, Tasseron S, Vermeulen JT, de Jonge N, et al. Fractionated electrograms in dilated cardiomyopathy: origin and relation to abnormal conduction. J Am Coll Cardiol. 1996; 27(5):1071-1078. [PubMed: 8609323]

5. Anderson KP, Walker R, Urie P, Ershler PR, Lux RL, Karwandee SV. Myocardial electrical propagation in patients with idiopathic dilated cardiomyopathy. J Clin Invest. 1993; 92(1):122-140. [PubMed: 8325977]

6. Gepstein L, Hayam G, Ben-Haim SA. A novel method for nonfluoroscopic catheter-based electroanatomical mapping of the heart. In vitro and in vivo accuracy results. Circulation. 1997; 95(6):1611-1622. [PubMed: 9118532] 
7. Hsia HH, Callans DJ, Marchlinski FE. Characterization of endocardial electrophysiological substrate in patients with nonischemic cardiomyopathy and monomorphic ventricular tachycardia. Circulation. 2003; 108(6):704-710. [PubMed: 12885746]

8. Soejima K, Stevenson WG, Sapp JL, Selwyn AP, Couper G, Epstein LM. Endocardial and epicardial radiofrequency ablation of ventricular tachycardia associated with dilated cardiomyopathy: the importance of low-voltage scars. J Am Coll Cardiol. 2004; 43(10):1834-1842. [PubMed: 15145109]

9. Cano O, Hutchinson M, Lin D, Garcia F, Zado E, Bala R, et al. Electroanatomic substrate and ablation outcome for suspected epicardial ventricular tachycardia in left ventricular nonischemic cardiomyopathy. J Am Coll Cardiol. 2009; 54(9):799-808. [PubMed: 19695457]

10. Yokokawa M, Good E, Crawford T, Jongnarangsin K, Chugh A, Pelosi F Jr, et al. Ventricular tachycardia originating from the aortic sinus cusp in patients with idiopathic dilated cardiomyopathy. Heart Rhythm. 2011; 8(3):357-360. [PubMed: 21056120]

11. Haqqani HM, Tschabrunn CM, Tzou WS, Dixit S, Cooper JM, Riley MP, et al. Isolated septal substrate for ventricular tachycardia in nonischemic dilated cardiomyopathy: Incidence, characterization, and implications. Heart Rhythm. 2011

12. Cesario DA, Vaseghi M, Boyle NG, Fishbein MC, Valderrabano M, Narasimhan C, et al. Value of high-density endocardial and epicardial mapping for catheter ablation of hemodynamically unstable ventricular tachycardia. Heart Rhythm. 2006; 3(1):1-10. [PubMed: 16399044]

13. Nakahara S, Tung R, Ramirez RJ, Michowitz Y, Vaseghi M, Buch E, et al. Characterization of the arrhythmogenic substrate in ischemic and nonischemic cardiomyopathy implications for catheter ablation of hemodynamically unstable ventricular tachycardia. J Am Coll Cardiol. 2010; 55(21): 2355-2365. [PubMed: 20488307]

14. Kuhne M, Abrams G, Sarrazin JF, Crawford T, Good E, Chugh A, et al. Isolated potentials and pace-mapping as guides for ablation of ventricular tachycardia in various types of nonischemic cardiomyopathy. J Cardiovasc Electrophysiol. 2010; 21(9):1017-1023. [PubMed: 20384656]

15. Tzou W, Zado E, Lin D, et al. Sinus rhythm ECG criteia assoicated with basal-lateral ventricular tachycardia substrate in patients with nonischemic cardiomyopathy. J Cardiovasc Electrophysiol. 2011; 22(12):1351-1358. [PubMed: 21736660]

16. Berruezo A, Mont L, Nava S, Chueca E, Bartholomay E, Brugada J. Electrocardiographic recognition of the epicardial origin of ventricular tachycardias. Circulation. 2004; 109(15):18421847. [PubMed: 15078793]

17. Daniels DV, Lu YY, Morton JB, Santucci PA, Akar JG, Green A, et al. Idiopathic epicardial left ventricular tachycardia originating remote from the sinus of Valsalva: electrophysiological characteristics, catheter ablation, and identification from the 12-lead electrocardiogram. Circulation. 2006; 113(13):1659-1666. [PubMed: 16567566]

18. Bazan V, Gerstenfeld EP, Garcia FC, Bala R, Rivas N, Dixit S, et al. Site-specific twelve-lead ECG features to identify an epicardial origin for left ventricular tachycardia in the absence of myocardial infarction. Heart Rhythm. 2007; 4(11):1403-1410. [PubMed: 17954399]

19. Valles E, Bazan V, Marchlinski FE. ECG criteria to identify epicardial ventricular tachycardia in nonischemic cardiomyopathy. Circ Arrhythm Electrophysiol. 2010; 3(1):63-71. [PubMed: 20008307]

20. Nazarian S, Bluemke DA, Lardo AC, Zviman MM, Watkins SP, Dickfeld TL, et al. Magnetic resonance assessment of the substrate for inducible ventricular tachycardia in nonischemic cardiomyopathy. Circulation. 2005; 112(18):2821-2825. [PubMed: 16267255]

21. Assomull RG, Prasad SK, Lyne J, Smith G, Burman ED, Khan M, et al. Cardiovascular magnetic resonance, fibrosis, and prognosis in dilated cardiomyopathy. J Am Coll Cardiol. 2006; 48(10): 1977-1985. [PubMed: 17112987]

22. Bogun FM, Desjardins B, Good E, Gupta S, Crawford T, Oral H, et al. Delayed-enhanced magnetic resonance imaging in nonischemic cardiomyopathy: utility for identifying the ventricular arrhythmia substrate. J Am Coll Cardiol. 2009; 53(13):1138-1145. [PubMed: 19324259]

23. Bala R, Ren J, Hutchinson M, et al. Assessing epicardial substrate using intracardiac echocardiography during VT ablation. Circ Arrhythm Electrophysiol. 2011; 4(5):667-673. [PubMed: 21880675] 
24. Hutchinson MD, Gerstenfeld EP, Desjardins B, Bala R, Riley MP, Garcia FC, et al. Endocardial unipolar voltage mapping to detect epicardial ventricular tachycardia substrate in patients with nonischemic left ventricular cardiomyopathy. Circ Arrhythm Electrophysiol. 2011; 4(1):49-55. [PubMed: 21131557] This study described the role of endocardial unipolar voltage mapping to predict an epicardial substrate in NICM patients

25. Polin GM, Haqqani H, Tzou W, Hutchinson MD, Garcia FC, Callans DJ, et al. Endocardial unipolar voltage mapping to identify epicardial substrate in arrhythmogenic right ventricular cardiomyopathy/dysplasia. Heart Rhythm. 2011; 8(1):76-83. [PubMed: 20933099]

26. Anter E, Hutchinson M, Deo R, et al. Surgical Ablation of Refractory Ventricular Tachycardia in Patients with Nonischemic Cardiomyopathy. Circulation: Arrhythmia Electrophysiology. 2011; 4:494-500. [PubMed: 21673018]

27. Sacher F, Roberts-Thomson K, Maury P, Tedrow U, Nault I, Steven D, et al. Epicardial ventricular tachycardia ablation a multicenter safety study. J Am Coll Cardiol. 2010; 55(21):2366-2372. [PubMed: 20488308] This study large multi-center study assessed the efficacy and safety of percutaneous epicardial access

28. Koruth JS, d'Avila A. Management of Hemopericardium related to Percutaneous Epicardial Access, Mapping and Ablation. Heart Rhythm. 2011

29. Fan R, Cano O, Ho SY, Bala R, Callans DJ, Dixit S, et al. Characterization of the phrenic nerve course within the epicardial substrate of patients with nonischemic cardiomyopathy and ventricular tachycardia. Heart Rhythm. 2009; 6(1):59-64. [PubMed: 19121801]

30. Bourke T, Vaseghi M, Michowitz Y, Sankhla V, Shah M, Swapna N, et al. Neuraxial modulation for refractory ventricular arrhythmias: value of thoracic epidural anesthesia and surgical left cardiac sympathetic denervation. Circulation. 2010; 121(21):2255-2262. [PubMed: 20479150] This study assessed the role of thoracic epidural anesthesia and/or surgical left cardiac sympathetic denervation for recurrent VT

31. Marchlinski FE, Callans DJ, Gottlieb CD, Zado E. Linear ablation lesions for control of unmappable ventricular tachycardia in patients with ischemic and nonischemic cardiomyopathy. Circulation. 2000; 101(11):1288-1296. [PubMed: 10725289]

32. Schmidt B, Chun KR, Baensch D, Antz M, Koektuerk B, Tilz RR, et al. Catheter ablation for ventricular tachycardia after failed endocardial ablation: epicardial substrate or inappropriate endocardial ablation? Heart Rhythm. 2010; 7(12):1746-1752. [PubMed: 20709191]

33. Vergara P, Trevisi N, Ricco A, et al. Late Potentials Abolition as an Additional Technique for Reduction of Arrhythmia Recurrence in Scar Related Ventricular Tachycardia Ablation. J Cardiovasc Electrophysiol. In Press. 


\section{Endocardial and Epicardial Voltage Map}

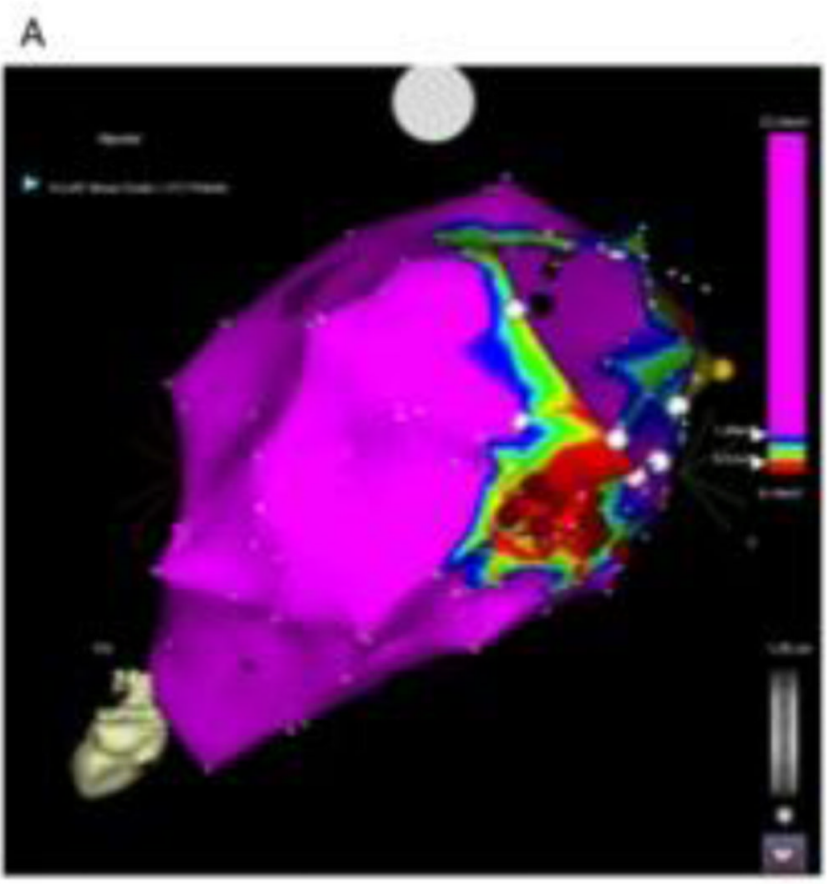

Endocardial Map

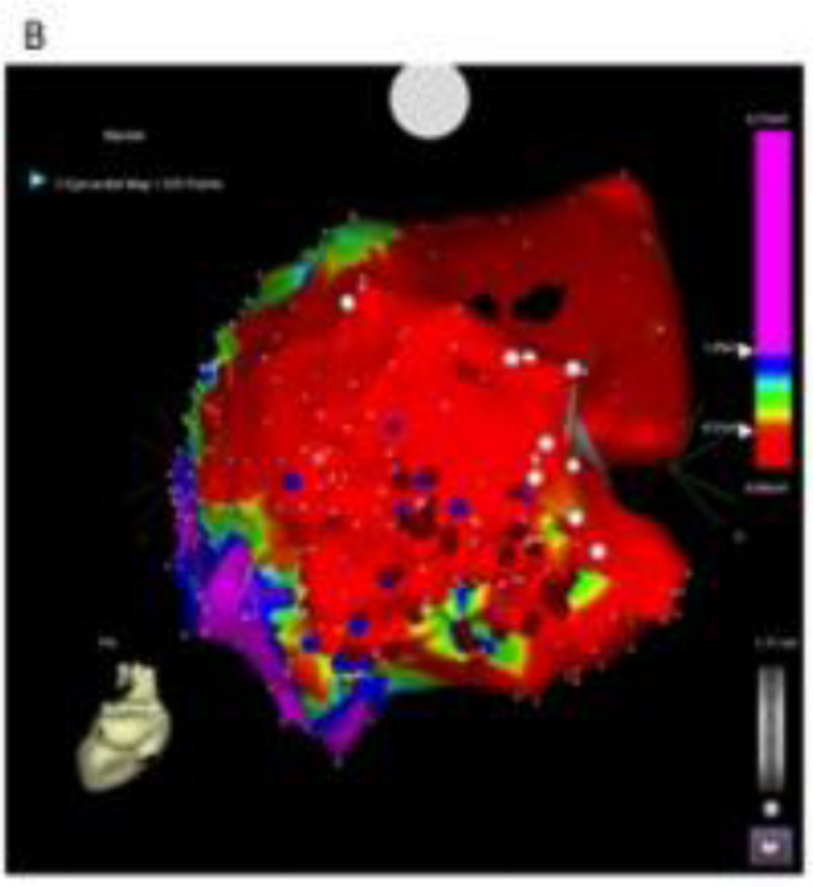

Epicardial Map

Figure 1.

Electroanatomic map of a patient with NICM undergoing VT ablation. Regions of scar $(\$ 0.5 \mathrm{mV})$ are denoted in red. The $1 \mathrm{~A})$ endocardial surface and $1 \mathrm{~B})$ epicardial surface are shown which show a greater area of scar on the epicardial surface. 


\begin{tabular}{|c|c|c|c|c|c|c|c|c|c|c|c|}
\hline & 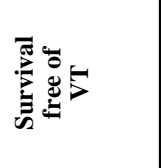 & $\begin{array}{l}\stackrel{0}{i} \\
i n\end{array}$ & ì & 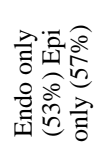 & 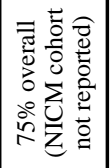 & $\frac{\stackrel{0}{2}}{2}$ & $\stackrel{\circ}{n}$ & 今̊ & 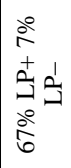 & $\stackrel{\circ}{\circ}$ & \begin{tabular}{|l|}
0 \\
$\infty$ \\
$\infty$
\end{tabular} \\
\hline & 尽高 & $\infty$ & ส & 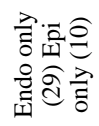 & $\simeq$ & 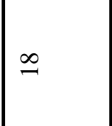 & $\simeq$ & \pm & $\stackrel{\infty}{-}$ & ¿ి & 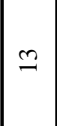 \\
\hline & 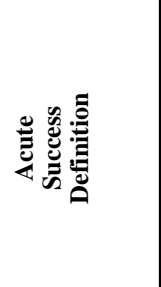 & 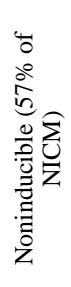 & 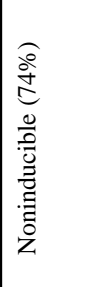 & $\begin{array}{l}\frac{0}{2} \\
\frac{0}{d} \\
\frac{0}{0} \\
\frac{0}{0} \\
\frac{0}{0} \\
\text { 言 } \\
\text { z }\end{array}$ & 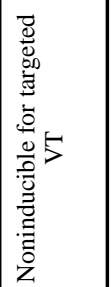 & 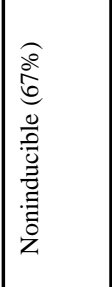 & 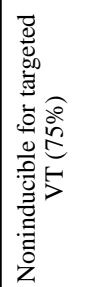 & 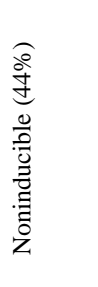 & 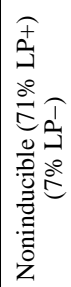 & 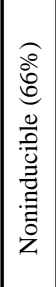 & 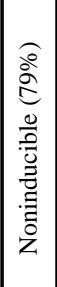 \\
\hline & 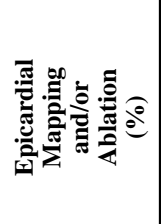 & 0 & 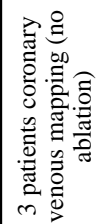 & 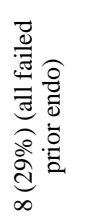 & 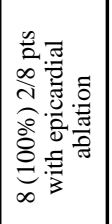 & 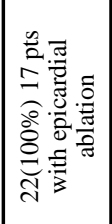 & 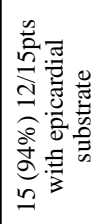 & 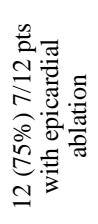 & $\begin{array}{l}\text { ồ } \\
\text { dè } \\
\text { r }\end{array}$ & 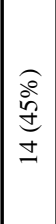 & $\stackrel{2}{2}$ \\
\hline$\sum$ & ن் & 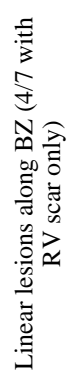 & 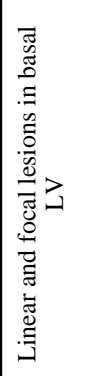 & 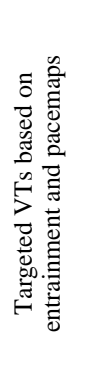 & 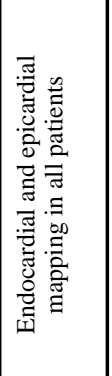 & 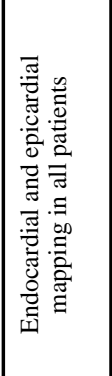 & 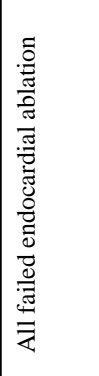 & 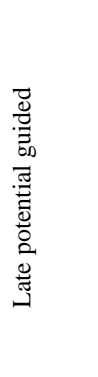 & 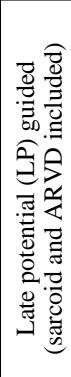 & 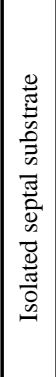 & 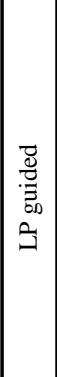 \\
\hline $\begin{array}{l}3 \\
: \\
3 \\
0 \\
0 \\
0 \\
0\end{array}$ & 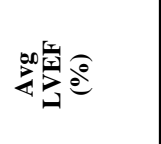 & $\forall$ & $\dot{m}$ & 崩 & mे & ¿ & లె & $\grave{\lambda}$ & 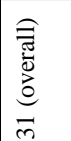 & in & $\ddot{m}$ \\
\hline 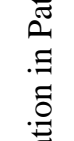 & 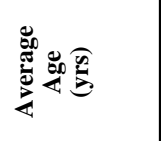 & f & $\bar{\sigma}$ & in & in & in & in & in & 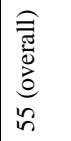 & in & 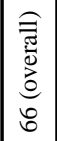 \\
\hline \begin{tabular}{l}
0 \\
\multirow{2}{5}{} \\
5 \\
5 \\
0
\end{tabular} & 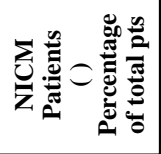 & $\begin{array}{l}\frac{o}{8} \\
\frac{8}{r}\end{array}$ & $\cong$ & i & $\begin{array}{l}\widehat{\sigma} \\
\text { ò } \\
\infty\end{array}$ & a & $\begin{array}{l}0 \\
\stackrel{0}{0} \\
\stackrel{d}{0} \\
0\end{array}$ & 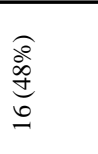 & 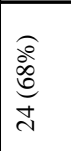 & $\vec{m}$ & 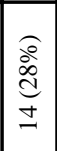 \\
\hline ڤ్ & こ్ & ঃ & ¿̊̀ & 营 & 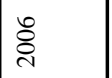 & 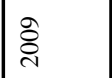 & 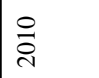 & 움 & $\stackrel{\circ}{\stackrel{4}{*}}$ & $\overline{\vec{c}}$ & $\frac{\text { กे }}{3}$ \\
\hline 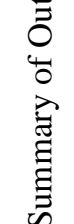 & 高 & 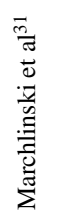 & 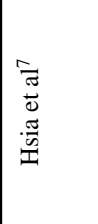 & 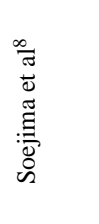 & $\begin{array}{l}\frac{1}{\pi} \\
\overline{0} \\
0 \\
0 \\
0 \\
0 \\
0 \\
0 \\
0\end{array}$ & 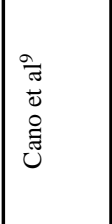 & 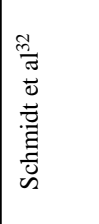 & 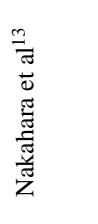 & 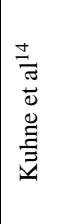 & 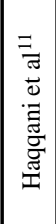 & 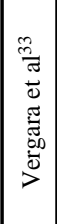 \\
\hline
\end{tabular}

